Meta

Journal des traducteurs

Translators' Journal

\title{
1492-1992 Voyage sur la mer océane : traduire et dévoiler
}

\section{Jean-Louis Cordonnier}

Volume 37, numéro 2, juin 1992

URI : https://id.erudit.org/iderudit/002371ar

DOI : https://doi.org/10.7202/002371ar

Aller au sommaire du numéro

Éditeur(s)

Les Presses de l'Université de Montréal

ISSN

0026-0452 (imprimé)

1492-1421 (numérique)

Découvrir la revue

Citer cet article

Cordonnier, J.-L. (1992). 1492-1992 Voyage sur la mer océane : traduire et dévoiler. Meta, 37(2), 179-192. https://doi.org/10.7202/002371ar d'utilisation que vous pouvez consulter en ligne.

https://apropos.erudit.org/fr/usagers/politique-dutilisation/ 


\title{
1492-1992 VOYAGE SUR LA MER OCÉANE: TRADUIRE ET DEVOILER
}

JEAN-LOUIS CORDONNIER

Université de Franche-Comté, Besançon, France

\begin{abstract}
Et si c'était cela, l'Europe, l'ouverture à une histoire décentrée pour laquelle le changement de cap, le rapport à l'autre cap ou à l'autre du cap est ressenti comme toujours possible? Ouverture et non-exclusion dont l'Europe aurait en quelque sorte la responsabilité ?
\end{abstract}

Jacques Derrida, «L'autre cap. Mémoires, réponses et responsabilités», Liber, no 5, Le Monde, 29 sept. 1990 , pp. 11-13.

La Harpe et l'ombre est un roman de Alejo Carpentier qui propose une version latino-américaine du personnage et du mythe de Christophe Colon ${ }^{1}$. Puisqu'il concerne la découverte de l'Amérique, ce roman doit revenir au premier plan de l'actualité pour le cinq centième anniversaire de la Découverte. Ce retour sous le feu des projecteurs n'est pas un retour comme les autres. Il a valeur de symbole. D'abord dans l'histoire. La conquête de l'Amérique est en effet, à une si vaste échelle, l'événement le plus important de l'histoire de l'humanité où deux cultures radicalement différentes se trouvent confrontées du jour au lendemain. Nous avons là une mine d'informations permettant d'étudier le comportement de l'homme occidental face à l'Autre. Ce roman a aussi valeur de symbole pour le présent. Parce qu'en cette époque d'internationalisation croissante des échanges, nous devons redéfinir notre rapport à l'Étranger. Et parce que Carpentier, au moyen de la littérature, veut que 1'Amérique s'approprie son propre mythe de Colon. Cela pose inévitablement le problème de la traduction. $\grave{A}$ travers elle, nous définissons notre rapport d'altérité face à l'Autre, le Latino-Américain. La traduction étant au cœur des rapports d'altérité, les modes de traduire sont concernés au premier chef.

\section{NÉCESSITÉ DE LA CRITIQUE}

Le rôle de la critique du traduire est de rompre avec cette vieillerie qu'est l'empirisme ethnocentrique et de lui opposer la traduction-dévoilement. La critique remet en cause l'ancestralité. Elle dérange le confort des habitudes, elle situe les limites et les incohérences de l'empirisme. Elle montre ce que doivent être les modes de traduire aujourd' hui et ce qu'ils seront demain. La critique participe à cette tâche exaltante, et dont nous avons la responsabilité historique, de façonnement de l'Homme décentré. Ce qu'elle met en évidence dans la traduction à venir, c'est le rôle social et culturel de celleci. Rôle qui est aussi historique. Ce qui signifie qu'il n'est pas figé, comme s'il était hors du temps et de la vie, mais qu'il change. La traduction, en effet, a fait la nation. Désormais, elle doit faire le monde. Pour cela, elle met en lumière l'Étranger dans toute sa clarté. Ce que ne fait pas précisément l'ethnocentrisme, qui, comme nous allons le démontrer, nationalise et désécrit ${ }^{2}$. 
Tout discours est unique et ne se donne qu'une fois. S'il est répété, il n'est déjà plus le même, par le fait que les conditions de sa production ont changé. C'est pourquoi on dit que les textes vieillissent. Lire Montesquieu aujourd'hui, même s'il redevient d'une certaine façon d'actualité, n'équivaut pas à lire cet auteur de son vivant. Mais le texteMontesquieu, lui, reste. Et c'est ce texte, dans son fonctionnement, dans ce qu'il est le sujet Montesquieu, qu'une traduction, aujourd'hui, aurait à montrer. Une telle visée du traduire engage bien d'autres compétences que celle de la simple connaissance des langues, cadre étriqué dans lequel se complaît la tradition. L'argument du seul bilinguisme qui sert à justifier la compétence en traduction est pernicieux, car il maintient la pratique du traduire dans l'empirisme, hors de la réflexion, et dans la problématique de la superposition mythique des langues, dans l'équivalence et dans le primat du sens. Nous suivrons plutôt Meschonnic :

Traduire ne peut pas éviter d'impliquer une théorie du discours. Selon qu'on traduit du sens ou de la signifiance, on découvre la théorie de la littérature qu'on met en ouvre, on se situe, on se date. La signifiance est une rythmique et une prosodie par lesquelles passe tout ce qui fait sens, et qui déborde la circonscription traditionnelle du sens, ses niveaux linguistiques. C'est l' enjeu majeur d'une critique de la traduction ${ }^{3}$.

La critique que nous allons mener ici porte sur la traduction d'un roman de l'écrivain cubain Alejo Carpentier : El arpa y la sombra, publié en 1979. La même année a paru sa traduction La Harpe et l'ombre, de René L. F. Durand. Nous trouvons dans ce roman un personnage: Christophe Colon. Mais il ne s'agit pas cette fois du personnage historique. Il s'agit d'un Colon carpentiérien qui est la démythification de la perception que nous en avons traditionnellement en Europe, et en même temps qui en constitue une re-mythification américaine. C'est pourquoi l'auteur cubain fait subir à Colon l'épreuve $d u$ texte. Démythification et re-mythification passent par une organisation du texte en tant que système. Par le fait qu'il est discours et unique. Qu'il a une valeur de discours qui transparaît dans la signifiance. Car, si l'on veut montrer le Colon carpentiérien, c'est bien le mode de signifier, par où passe tout le sens, qu'il convient de traduire.

Et c'est la raison pour laquelle un traducteur, par rapport au texte, doit avoir ce que nous appelons un projet de traduction. Cela signifie que le système du texte a été repéré et que lui correspondra un système du texte dans la traduction. Non pas au niveau de la langue, mais dans le discours. Ce dernier est travail de la langue de traduction. Il emprunte et donne à voir l'étrangeté et l'étrangéité du texte produit par le sujet énonciateur. Dans la traduction de El arpa y la sombra, on devrait retrouver une désécriture de l'histoire qui démythifie et qui re-mythifie. Un Christophe Colon qui redescend de son haut piédestal et qui se voit doté d'un versant latino-américain. Mais avant d'examiner la traduction de El arpa y la sombra, arrêtons-nous un instant sur les conceptions que se fait $\mathrm{A}$. Carpentier du rôle de l'écrivain dans l'histoire, à travers l'identité latino-américaine, le baroque et le réel-merveilleux.

\section{UNE ÉCRITURE POUR MANIFESTER L'IDENTITÉ}

Pour Carpentier, il y a une grande différence entre 1'Européen et le LatinoAméricain nés au début de ce siècle. Le premier a grandi dans des cités chargées d'histoire et qui témoignent d'un lent processus dont on peut remonter le fil jusqu'aux origines de la civilisation occidentale. Tandis que le second a vécu dans des villes de béton qui se sont mises à pousser d'un seul coup, au milieu des révolutions, la société connaissant de profondes mutations alors que jusque-là elle avait plutôt tendance à stagner, depuis le $X V I^{\mathrm{e}}$ siècle. Devant tous ces bouleversements, la question à laquelle est fatalement conduit le Latino-Américain a un triple aspect: connaître les origines, 
entrevoir le devenir de la société et, première tâche entre toutes, répondre au "Qui suisje?" (Quién soy yo ${ }^{4}$ ?). À ce propos, l'une des particularités marquantes du continent sud-américain, c'est la rencontre unique et historique, sur le même territoire, entre les trois cultures : noire, amérindienne et européenne. Rencontre qui est à l'origine de la plus extraordinaire symbiose, du plus extraordinaire métissage que l'on ait vus jusqu'à présent. De là l'originalité de l'Amérique latine. Carpentier pense que l'histoire du continent doit être appréhendée dans son unité «pour finir de comprendre réellement $c e$ que nous sommes, et quel est le rôle que nous devrons jouer dans la réalité qui nous entoure et qui donne un sens à nos destins ${ }^{5}$ ». D'où le rôle social de l'écrivain latinoaméricain, dont l'une des tâches justement est de mener cette quête. Ce que personne ne fera à sa place. Et Carpentier cite à ce propos la formule de José Martí, qui disait déjà en 1893: «Ni le livre européen, ni le livre yankee ne nous donneront la clé de l'énigme hispano-américaine ${ }^{6 . » ~ I l ~ s ' a g i t ~ d o n c, ~ p a r ~ l ' e ́ c r i t u r e, ~ d e ~ f a i r e ~ a f f l e u r e r ~ u n e ~ c o n s c i e n c e ~}$ continentale et, en cherchant les racines, de dire l'américanéité.

Dans ces conditions, il n'est pas du tout surprenant que Carpentier en vienne à s'intéresser de près à la figure de Cristophe Colon, qui lui aussi s'est trouvé en proie à la frénésie de la nomination, dès lors qu'il n'entrevoyait pas celle, déjà existante, des peuples précolombiens. L'écrivain se donne donc pour tâche de montrer l'américanéité par une profonde connaissance de son histoire et de sa culture, mais aussi par le travail sur le langage. Et l'un des moyens d'expression qui convient particulièrement bien à la réalisation de cet objectif, c'est le roman. Pour Carpentier, si le roman peut être en crise, c'est parce qu'il reste inféodé aux vieux schémas. Au contraire, le roman ne peut que se trouver en bonne santé s'il cherche l'élément épique. Le roman a donc, à travers ses personnages qui traversent les grandes mutations et les grands bouleversements américains, une dimension collective. La réalité de l'Amérique est si riche que le narrateur se trouve face à une quantité infinie d'éléments constitutifs de l'epos. Toute l'œuvre de Carpentier en est imprégnée. Comme dans La Harpe et l'ombre, où Colon est un personnage épique s'il en est. D'ailleurs, l'épique ne traverse pas seulement le passé, mais aussi le présent.

Passé, présent et futur sont donc pour Carpentier inextricablement mêlés. Le passé en effet est très présent dans le présent, et parallèlement on pressent tout le bouillonnement du futur latino-américain. Pour le montrer, il faut donc que l'écrivain brise la conception traditionnelle et linéaire du temps, pour pouvoir tirer le plus grand parti possible de cette réalité spécifique à l'Amérique du Sud. C'est ce qui se passe encore dans La Harpe et l'ombre, roman construit autour de trois plans bien distincts : le premier se situe dans la deuxième moitié du XIXe siècle et met en scène le pape Pie IX et sa décision d'enclencher la canonisation de Colon. Le deuxième est une rétrospective de la vie du Découvreur, par le Découvreur lui-même, sur son lit de mort. Quant au troisième plan, il se présente sous la forme d'une carnavalisation, typique dans la littérature baroque, et mêle tous les âges, de Colon à Paul Claudel. Il permet donc de confronter les personnages qui ont fait de l'Amiral un mythe, de détruire ce mythe, mais en même temps d'en donner une version latino-américaine. En conséquence, le roman doit être «synchronique».

Pour accéder à sa propre identité, et pour la communiquer à ses compatriotes du continent, l'écrivain se trouve donc devant le fait d'avoir à manifester par une écriture originale ces aspects de la réalité américaine qui sont, rappelons-le, l'élément épique, cette symbiose qu'est le métissage, la grandeur de la nature et l'interconnexion des plans historiques. Pour cela, il faut une langue. Il est donc dévolu au roman de «créer une langue» (crear un idioma ${ }^{7}$ ) qui soit propre à l'Amérique latine et qui se différencie de plus en plus de l'espagnol d'Espagne. Plus, la création d'un «langage» est l'un des 
aspects essentiels de l'œuvre de Carpentier, qui, en écoutant et en réexprimant la réalité, fait résonner, comme le remarque Carlos Fuentes, «ces pistes magnétiques [...], "ces sons bruts" $[\ldots]$ qui $[\ldots]$ constituent une nouvelle totalité narrative dans laquelle la fiction se fait elle-même au moyen d'un langage qui est réflexion sur le langage ${ }^{8}$ ». Ces «pistes magnétiques», ces «sons bruts» sont ceux du monde latino-américain que l'auteur veut donner à voir. Or, ce monde est, et depuis toujours, profondément et par essence, baroque. Dans ces conditions, la littérature, qui a pour rôle de l'interpréter, sera baroque elle aussi. Le caractère baroque de la littérature n'est donc pas seulement une affaire de volonté, mais il est encore inhérent aux modes d'être du continent sud-américain. Maintenant, il n'est pas inutile pour le traducteur de savoir ce que Carpentier entend quand il parle de «ce qui est baroque et de ce qui est réel-merveilleux ${ }^{9}$ ».

L'auteur s'emploie à combattre ce poncif tenace qui fait naître l'art baroque au $\mathrm{XVII}^{\mathrm{e}}$ siècle et qui le considère comme une sorte de maniérisme, une sorte de transition entre les genres. En revanche, Carpentier, se référant à Eugenio d'Ors, dont les écrits sur la question font autorité, affirme que le baroque au contraire est une constante de l'humanité, qu'il revient cycliquement dans l'histoire, qu'il concerne tous les arts et qu'il est une immense pulsion créatrice qui signale les sommets atteints par une culture. Il y a donc un esprit baroque qui traverse les siècles et les hommes, et Carpentier cite Alexandre, Charlemagne, Napoléon. Et dans la littérature, Quevedo, Calderón, Góngora, Arioste (Orlando furioso), les littératures hindoue et iranienne, Shakespeare, Proust et Rabelais, ce dernier profondément baroque, car il était «Inventeur de mots, enrichisseur

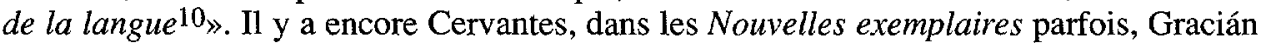
(toute la prose) et le romantisme, qui, pour Carpentier, est complètement baroque. De Delacroix à Wagner, de Lord Byron à Novalis et Goethe, de Rimbaud à Lautréamont. Le baroque est donc signe de richesse artistique et surgit habituellement dans les périodes de bouleversement, de mutations, où l'innovation se déploie tous azimuts.

Par contre, quand il y a stabilité et certitude, c'est l'académisme qui prend le dessus. Il y a alors grand respect des règles et des normes et, en conséquence, obstacle à la nouveauté. C'est ce qui se passe dans le classicisme. À ce propos, Carpentier ne se satisfait pas des définitions qu'en donnent les dictionnaires qui se contentent de renvoyer à l'Antiquité, et pour lui, le classicisme est d'abord un équilibre géométrique entre espaces vides et espaces remplis. Il donne alors pour exemple le Parthénon, où l'harmonie est créée entre colonnes et espaces vides, le palais de Versailles et l'Escorial. Dans ces trois cas, l'équilibre géométrique domine. À l'opposé, le baroque «se caractérise par l'horreur du vide, de la surface nue, de l'harmonie linéaire géométrique, style où autour de l'axe central se multiplient ce que nous pourrions appeler les noyaux proliférants, c'est-à-dire des éléments décoratifs qui remplissent totalement l'espace occupé par la construction, par les murs, par tout l'espace architecturalement disponible, avec des motifs qui sont dotés d'une expansion propre et lancent, projettent les formes avec une force expansive vers l'extérieur. C'est-à-dire que $c$ ' est un art en mouvement, un art de pulsion, un art qui va du centre vers l'extérieur et qui rompt, d' une certaine façon, ses propres marges ${ }^{11}$.

La connaissance de ce passage est fondamentale pour appréhender l'écriture baroque de Carpentier. Dans La Harpe et l'ombre, on retrouve ce rôle joué par un noyau central et par des noyaux proliférants qui l'accompagnent, au niveau des phrases et de la structure même de l'œuvre. C'est ainsi que sur les trois chapitres, le deuxième est consacré au récit de la vie de Colon par lui-même, alors que les chapitres d'ouverture et de fermeture sont comme des excroissances au personnage et au mythe qui l'entoure. Dans le premier chapitre est montrée la tentative par le pape Pie IX et en France par l'Église ultramontaine de béatifier Christophe Colon, en espérant grâce à cette décision 
reprendre le contrôle des âmes d'une Amérique secouée par les mouvements d'indépendance et de révolution. Quant au dernier chapitre, il est aussi un noyau proliférant en ce sens qu'il détruit par la dérision le mythe occidental de l'Arrondisseur de la Planète, mais en même temps, il est fait place à un nouveau mythe, baroque et latino-américain, cette fois. On retrouve naturellement cette disposition au niveau de la phrase, qui comprend de nombreuses incidentes et où le sujet, le verbe, l'élément clé, occupent souvent une position centrale.

Pour Carpentier, l'Amérique a toujours été baroque. Il en veut pour preuve la littérature maya (Popol Vuh, Chilam Balam), la poésie nahuatl, l'architecture des temples aztèques où les vides n'existent pas, le temple de Mitla à côté de Oaxaca (Mexique), où les figures géométriques s'engendrent elles-mêmes dans un mouvement proliférant. Et si ni le gothique ni le roman ne se sont installés en Amérique, par contre, le plateresque, lui, a conquis le continent. Il s'est marié à l'inspiration artistique indienne pour donner des chefs-d'œuvre baroques, comme on en trouve au Mexique : églises de Tepotzotlan près de Mexico, et à côté de Cholula, de San Francisco Acatepec, et de Santa María Tonantzin, laquelle arbore une incroyable féerie métissée aux milliers de motifs aux tons pastel. En Amérique latine, la richesse du métissage accentue le phénomène baroque, chacun des éléments particuliers de cette immense symbiose venant grossir le fleuve baroque. Il faut donc prendre en compte ce que Carpentier appelle «la créolité» (la criollidad), qui est le carrefour où se croisent et se mêlent les trois cultures : l'indienne, la noire et la blanche. Cette rencontre culturelle a permis l'apparition de ce phénomène particulier qu'est le «réel-merveilleux».

Mais le réel-merveilleux n'est pas le «merveilleux» au sens habituel. Avec le temps, le mot s'est usé. On entend maintenant par merveilleux quelque chose qui suscite l'admiration par sa beauté et son caractère extraordinaire. Le mot a incontestablement une connotation positive. Il évoque une certaine douceur de vivre. Mais, pour Carpentier, le seul aspect qu'il y a à en retenir, c'est le côté «extraordinaire»: Tout ce qui est insolite, tout ce qui est étonnant, tout ce qui sort des normes établies est merveilleux 12 . Qu'un fait soit beau, cruel, admirable ou laid (cf. par exemple les contes de fées) importe peu. Mais il faut encore distinguer entre l'insolite qui a été prévu, prémédité, et qui n'est que «mystère fabriqué», comme c'est le cas par exemple dans le surréalisme, et l'insolite que Carpentier défend, et que l'on trouve en quelque sorte à l'état naturel en Amérique : «Ici l'insolite est quotidien, il a toujours été quotidien ${ }^{13}$.» Le réel-merveilleux, c'est donc la réalité du continent dans son ensemble et à tous les niveaux. Il y a par conséquent un travail à réaliser sur le langage pour montrer l'insolite latino-américain. La langue espagnole n'était pas préparée à dire cette nouvelle réalité. Et l'on sait que Colon, le premier, et plus tard Cortés se sont trouvés désemparés, en présence d'un monde entièrement nouveau, pour nommer les choses. Or, cinq siècles après la prise de possession de l'Amérique par le Grand Navigateur, la tâche de la nomination est toujours à l'ordre du jour. L'Amérique latine a à s'approprier un langage qui soit le sien propre. Le rôle de l'écrivain est donc de produire du langage qui traduise la réalité baroque et réellemerveilleuse de l'Amérique. Le castillan se prête particulièrement bien à ce travail, car il

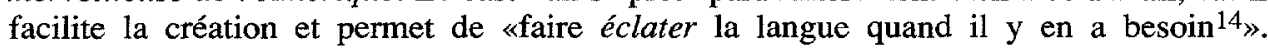
Carpentier se pose donc en interprète de son monde, et l'on sait qu'il y a consacré toute son œuvre. Traduire Carpentier, c'est donc a fortiori traduire son travail sur le langage et, partant, c'est traduire le baroque et le réel-merveilleux qu'il place sous nos yeux. Toute traduction qui détruit l'écriture carpentiérienne vide le monde latino-américain de sa substance, le met à plat, le rationalise, l'annexe. En un mot, elle le prive de son être. 


\section{LA HARPE ET L'OMBRE OU LA RECHERCHE DES ORIGINES}

C'est Christophe Colon qui est à l'origine du métissage de la société latinoaméricaine moderne et de sa profonde originalité. Notre auteur pense en effet que la Découverte représente une véritable coupure dans l'histoire mondiale: «c'est l'événement le plus important de l'histoire. Parce qu'il existe dans l'histoire universelle un homme antérieur à la découverte de l'Amérique, et un homme postérieur ${ }^{15}$. D'un autre côté, Colon est le premier métis européo-américain. Dans le mythe, c'est la facette européenne qui domine largement. Mais l'Amérique a besoin de découvrir sa propre version. C'est pourquoi Carpentier a écrit La Harpe et l'ombre, où le mythe montrera cette fois la facette américaine d'un Colon désormais américano-européen 16.

C'est.bien le Découvreur qui est le sujet et le pourquoi du roman. Et non, contrairement à ce qui a pu être dit ${ }^{17}$, ce pape réactionnaire qu'était Pie IX qui était le souci majeur de l'auteur. On se souvient que le centre constitue une position essentielle de l'œuvre baroque. Nous avons déjà indiqué que des trois chapitres qui composent le roman, le chapitre central est consacré à Colon proprement dit. Et ce n'est pas tout. Car ce cœur de l'œuvre, comme le remarque judicieusement Jacqueline Tauzin ${ }^{18}$, se trouve à son tour divisé en treize parties, dont le noyau central est lui-même chargé de signification : il est en effet consacré au Nouveau Monde, à la Découverte, précisément, qui se trouve donc être au centre exact du roman. Il n'y a donc aucun doute sur les intentions de Carpentier.

On connaît l'intérêt que l'auteur portait aux épigraphes. Le titre du roman, justement, provient d'une épigraphe, placée en tête du roman, et tirée de la Légende dorée:

Dans la harpe, quand elle résonne, il y a trois choses: l'art, la main et la corde.

Dans l'homme: le corps, l'âme et l'ombre ${ }^{19}$.

Les trois parties du roman s'intitulent respectivement: «La harpe», «La main» et «L'ombre». On remarque immédiatement deux choses. Que le titre du roman se compose des deux termes qui encadrent l'épigraphe. Et que la «main», qui est le cœur du roman, n'a pas été reprise dans le titre. Cette absence nous indique que la vie de Colon dans l'Histoire n'est pas proprement l'objet du livre. Il ne s'agit pas en effet de «raconter les choses comme elles se déroulèrent, mais comme elles durent ou purent se dérouler ${ }^{20} \gg$. La «main» étant au cœur de la question, c'est à un équilibre entre la «harpe» et l'«ombre» que nous convie Carpentier. Mais la «main», c'est d'abord celle de Colon, qui tient la barre de sa caravelle, la Santa María, et qui par ce geste tend le fil qui va réunir à jamais les deux continents. Ce fil tendu par un marin du vieux continent en dit long sur l'assujettissement que l'Amérique va dès lors connaître. Si l'Amérique d'aujourd'hui se cherche un Colon pour son panthéon, ce n'est assurément pas de son côté assujetisseur qu'elle a besoin. Non pas qu'il s'agisse pour autant de renier quoi que ce soit, car au contraire l'Amérique est à la recherche de ses origines, mais il est question plutôt de façonner un Colon baroque et dans lequel elle pourra puiser son identité. La «main», c'est donc celle du Découvreur qui écrit l'histoire, au sens propre et au sens figuré. Mais c'est aussi, dans le double jeu trouble de la création, celle de Carpentier qui à son tour écrit, mais cette fois sur l'histoire.

Si l'on se reporte à l'épigraphe, on remarquera que la «main» fait partie de la «harpe». Qui est aussi l'art et, dirons-nous, la littérature, par conséquent. Et il y a dans «La harpe» un personnage qui aimerait bien que la «main», c'est-à-dire Colon, pince à nouveau les cordes sur la partition de l'histoire. Ce personnage, c'est Pie IX (pape de 1846 à 1878), et connu pour les positions radicales qu'il avait prises dans l'encyclique Quanta cura et dans le Syllabus contre le libéralisme et le socialisme. En signant le décret 
qui engageait la postulation de Colon pour la canonisation, il cédait aux pressions d'une Eglise conservatrice inquiète devant la vague des révolutions et des guerres d'indépendance qui secouaient tout le continent sud-américain. Il est donc question de faire de Colon un saint, de ranimer son image, en quelque sorte, en espérant que la canonisation donnera l'envie à l'Amérique de rester ou de revenir dans le giron de Rome. Parallèlement s'était développée toute une littérature hagiographique, aujourd'hui tombée dans 1'oubli, idéalisant le personnage du Grand Amiral. A commencer par Roselly de Lorgues, spécialiste de l'apologie de Colon, qui a écrit un Christophe Colomb (1856) et qui a été chargé par la reine d'Espagne en 1893 de transmettre au Saint Père sa demande de béatification du Grand Voyageur. Il y a ensuite ces deux ouvrages de Léon Bloy: Le Révélateur du Globe (1883), et Christophe Colomb devant les taureaux (1890), que Carpentier qualifie d' «incroyables», tant leur outrance l'a surpris. La «harpe», c'est donc le jeu qui consiste à faire renaître Colon de ses cendres, à le mythifier d'une certaine manière, à des fins politiques. Et c'est là que réside aussi, non pas l'axe du roman, mais l'une de ses raisons d'être : renverser ce mythe qui s'est peu à peu constitué en Occident à coups d'hagiographies, retrouver au-dessus de ces textes l'ombre de Colon pour que puisse vibrer la corde latino-américaine, cet autre élément de la «harpe», à côté de l' "art» et de la «main», et mise entre parenthèses par l'auteur. Travail d'archéologie.

C'est donc l'«ombre» qui plane sur le troisième et dernier chapitre. Et c'est le seul élément parmi les trois qui composent l'homme que Carpentier retient. En effet, dans l'épigraphe apparaissaient également le «corps» et l'«âme». Mais ceux-ci sont les victimes du temps. Le «corps» est devenu poussière. Quant à l'«âme», elle ne relève pas de la préoccupation ni du ressort de Carpentier, humain parmi les humains. Mais ce qui intéresse ce dernier, c'est l'«ombre» de Colon. Car l'Amérique n'a, à vrai dire, pas besoin $\mathrm{du}$ «corps» de Colon. Mais elle a besoin de son «ombre». Ou plutôt d'une «ombre», car celle-ci a pour qualité première d'être fugitive et changeante. Tout dépend en effet de l'orientation de la lumière. Carpentier s'évertue à détruire l'«ombre» qui apparaît sous l'éclairage européen. Là, la «harpe» joue faux car il y a manipulation, falsification de 1'histoire à des fins partisanes. La «main» montre un Colon qui, s'il est grand, se montre aussi cupide et menteur, autrement dit plus proche des réalités terrestres. Et c'est la carnavalisation, si caractéristique de la littérature baroque, qui met en miettes définitivement l'«ombre» irréelle tissée sur le vieux continent. Et de la confrontation entre la «harpe» et l'«ombre», sort une nouvelle partition littéraire baroque qui, pour la première fois, depuis que le Révélateur du Globe a mis le pied en Amérique, correspond à la réalité de ce continent, à son identité et à ses attentes. Non pas écrire l'histoire, mais écrire le mythe. Pour que la «harpe» joue juste.

Ainsi, la «harpe», ou encore la littérature, est en harmonie avec l'«ombre» et avec la réalité latino-américaine, et celle-ci est prise dans une œuvre de sacralisation, ce que remarque aussi Claude Fell: «Il faudra attendre, pour que l'Amérique devienne une "idée" que le temps - et la littérature - la sacralisent ${ }^{21}$.» Or, toute l'auvre de Carpentier participe de ce travail de sacralisation. $D$ 'où, dirons-nous, une responsabilité de plus pour le traducteur. D'autant plus que La Harpe et l'ombre est en plein au creux des rapports d'altérité. Ce roman posant l'américanéité, celle-ci naturellement doit apparaître telle quelle, baroque et réelle-merveilleuse dans la traduction. Car il faut bien accepter d'abord, et faire entrevoir ensuite, une voix différente de la nôtre.

\section{TRADUCTION ET SIGNIFIANCE}

Traduire ne peut se faire en maintenant dans l'ombre la vérité de l'Autre. Il s'agit là d'un choix librement consenti, éthique et politique. La traduction montre le mode de 
traduire, et du coup la position du traducteur. Dans le cas qui nous occupe, il n'y a pas la littérature baroque et sa traduction, mais bien des textes baroques et leurs possibles traductions. On peut accorder le primat au sens et traduire de la langue. C'est-à-dire violenter le texte, le détruire en tant que système, pour qu'il se plie au sens et à la langue de traduction. On se situe alors, qu'on le veuille ou non, dans la langue de l'annexion. On peut au contraire tenter le pari de montrer la signifiance. Ce qui n'est pas une position contre le sens. Il n'y a pas - ce que dit une vision simpliste de la conception saussurienne du signe linguistique - d'un côté le sens, de l'autre la signifiance, et qui seraient irréductibles l'un à l'autre. Mais le sens traverse la signifiance de part en part, et à son tour celle-ci est productrice de sens. La traduction-annexion, aveuglée par la toutepuissance du sens, ne voit pas scintiller l'autre versant du texte, qui est la signifiance, précisément. Or, le dévoilement de l'Autre commence par la révélation du sujet-écrivain. Et celui-ci transparaît dans une écriture, dans son écriture, qui organise le texte et corrélativement fait naître du sens. Les enjeux se situent en effet au niveau du discours et de la littérature. Quel que soit le type de texte. Face à la pratique annexionniste, on oppose donc la prise en compte de la signifiance, lieu où se trouve «l'enjeu majeur d'une critique de la traduction».

À propos de La Harpe et l'ombre, il peut être de quelque intérêt de mesurer les écarts que Carpentier s'est permis avec l'Histoire, si l'on s'intéresse à l'Histoire ${ }^{22}$. Mais si l'on s'intéresse au roman, de telles considérations ne nous apportent rien sur le fond, du moins en vue de l'opération traduisante. Car l'auteur, il l'a dit, ne s'est pas donné pour but de faire cuuvre d'historien. Même si chez lui «on sent la bibliothèque et l'archive ${ }^{23}$ ». Ce n'est pas tant à l'homme Colon que Carpentier s'intéresse mais, le mythe n'appartenant plus désormais à la seule Europe, à ce qu'est devenu son personnage au cours de l'histoire et à ce qu'il doit devenir dans sa version latino-américaine. C'est cela que nous raconte La Harpe et l'ombre. Le roman est donc bien producteur de sens. Mais à aucun moment ce sens ne se donne à voir selon un mode explicite. Il passe tout entier par la signifiance. Qui est baroque et réelle-merveilleuse. Traduire l'essence du mythe cré par Carpentier, c'est donc traduire la signifiance du texte. Et c'est par rapport à elle que doit s'organiser le projet de traduction. Le texte carpentiérien n'est pas prisonnier de la langue. En tant que discours, il travaille aux confins de celle-ci. Tout en créant, il la bouscule et l'enrichit. C'est en effet en ce lieu que travaille la littérature, du moins quand elle se renouvelle. Et c'est en cet endroit aussi, aux marges de la langue, que doit opérer la traduction, si elle veut assumer sa tâche de re-création de l'œuvre. La re-création de $E l$ arpa y la sombra n'a en effet ni à se situer dans le langage académique, ni à suivre les critères de l'esthétique classique. Au contraire, il y a une antinomie radicale entre ceux-ci et le baroquisme fleuri, touffu et orfévré de Carpentier.

La traduction de Durand, malheureusement, plutôt que de tenir les valeurs du discours, ce qui aurait pour conséquence inévitable de heurter de plein fouet la langue sur ses frontières, préfère détruire le texte en se rangeant derrière la norme de la langue. On se rappelle le travail d'orfèvre que Carpentier veut produire sur le langage pour accéder aux origines de l'identité latino-américaine. Ce travail, la traduction de Durand le brouille et le défait. Partant, c'est tout le visage de l'Autre qui devient flou. Et c'est pour le faire briller en français que nous retravaillons à la retraduction de El arpa y la sombra. Mais une telle retraduction passe par la critique de la traduction existante qui n'est qu'une approche du texte original. Car son travail de détextualisation passe par une déformation du rythme, au sens où celui-ci, comme l'entend Meschonnic, est considéré «comme organisation de la parole dans l'écriture, socialité et subjectivité du discours 24 ». C'est toute une poétique, qui passe par une rythmique et une prosodie, que Carpentier met en place pour parvenir au but qu'il s'est donné en tant qu'écrivain. Le rythme, ce n'est pas 
seulement, comme on le pense généralement, la seule répétition de sons ou de lettres. Mais c'est un ensemble beaucoup plus vaste qui fait sens et qui prend en compte le fameux noyau central de la phrase baroque et les groupes proliférants alentour; c'est le rapport entre le texte carpentiérien et les très nombreuses citations, exactes ou non, mais d'abord et toujours en dialogue avec le texte ; c'est aussi le surgissement de l'oral dans l'écrit; l'ordre des mots et des groupes; la variété et la recherche du lexique; la ponctuation, notamment l'utilisation du tiret; la grande abondance et l'extrême diversité des répétitions, phénomène typique de l'art baroque; le recours fréquent à la majuscule, la formation abondante de noms propres à partir de vocables de la langue courante; c'est encore l'utilisation de l'italique pour faire ressortir; la rupture dans l'utilisation des temps, souvent quand transparaît l'oralité ; ou bien le mélange des registres, ou la recherche de musicalité. Tout cela crée un mouvement dans le texte, une gestuelle qui montre, pourrait-on dire, le corps latino-américain. Et c'est en considération de cet ensemble qu'on peut parler d'un texte comme système. C'est en cet endroit aussi que la traduction de Durand n'a pas de projet, au sens où nous l'avons défini. Se situant dans la langue, elle met obligatoirement à plat l'écriture carpentiérienne. N'apercevant pas, comme nous y avons déjà fait allusion, l'autre versant du texte, qui n'est pas si secret qu'on veut bien le croire, elle désorganise le noyau central et les groupes qui l'entourent, elle ne voit pas le dialogue intratextuel entre les citations et le texte proprement dit, elle désoralise, elle appauvrit la richesse lexicale et syntaxique; elle se comporte avec une extrême légèreté avec la ponctuation; des phrases baroques longues et touffues, elle fait des confettis ; mais ce n'est pas tout, le système des majuscules est démoli, et celui des répétitions subit la censure classique; il y a encore l'écart entre les registres qui est réduit, aplani, notamment par un rabotage du familier et du vulgaire; quant à la musicalité à laquelle Carpentier était particulièrement sensible, elle ressort anémiée du non-respect de la prosodie. Le respect de la langue n'explique pas tout. C'est une certaine idéologie de la littérature et de la traduction, c'est-à-dire une certaine idéologie du langage, qui explique une telle traduction. «L'idée que vous avez du langage est votre portrait» et dans «votre portrait en langage est signée une vision du monde», dit Meschonnic ${ }^{25}$. Même si elle l'ignore, cette pratique de la traduction se situe dans le droit fil de la tradition ethnocentrique, car c'est une annexion et une caricature de l'Autre que, sans en avoir l'air, elle nous soumet.

\section{ET LA TRADUCTION SORTIRA L'AUTRE DE LOMBRE}

On analysera ici un court extrait de El arpa y la sombra, tiré de «La main» ( $2^{\mathrm{e}}$ partie), où apparaissent d'une façon évidente à la fois le travail sur le langage et le travail sur le personnage de Colon. Les caravelles sont ancrées à proximité de la côte des Indes que l'on vient de découvrir. La première descente à terre, la «Prise de Possession», est prévue pour le lendemain. C'est la nuit. Colon, qui ne trouve pas le sommeil, à la veille d'un événement si excitant, ressasse sa vie. Au début du passage, il est question de Rodrigo de Triana, le marin qui a été le premier à crier: Terre ! On sait que Colon avait promis dix mille maravédis de récompense au premier de ses marins qui verrait la terre mais qu'il n'a pas tenu parole. Voici cet extrait:

En cuanto a su renta de diez mil maravedís (y esto sí habré de decirlo al confesor) podrá anotarlo en hielo — ! y cuidado no ande reclamando mucho o alborotando más de la cuenta, ya que le sé cosas que no le conviene que se sepan! -, porque esa renta me la he apropiado ya en beneficio de mi Beatriz, la guapa vizcaína de quien tengo un hijo sin haberla llevado al altar, y que, desde hace tiempo, en lágrimas padecía mi desapego y mi olvido - desapego y olvido debidos al Real Favor que sobre mi hubiera derramado, cual brotada de 
cornucopia romana, la fortuna de tres naves prestas a zarpar, con la confusión de mis enemigos, la embriaguez de nuevos rumbos, la gloria de estar aqui esta noche, esperando la salida de un sol que tarda, que tarda - i y cómo tarda, coño! - en asomar, y acaso la inmortalidad, en la memoria de los hombres, de Quien, salido de donde salí, podía aspirar ya al título de Ensanchador del Mundo...; No, Rodrigo! ; Te jodiste! Me quedo con tus diez mil maravedís de renta!... (p. 103).

Et voici la traduction de Durand :

Quant à sa rente de dix mille maravédis (et cela certes je devrai le dire au confesseur), il peut en faire son deuil. Et qu'il ne s'avise pas de trop réclamer, ou de faire le malin, car je sais sur lui des choses qu'il a intérêt à cacher! Cette rente, je me la suis appropriée au bénéfice de ma Beatriz, la belle Biscaïenne dont $j$ 'ai un fils sans l'avoir conduite à l'autel et qui depuis longtemps pleurait mon indifférence et mon oubli. Indifférence et oubli imputables à ma bonne fortune, qui, tel un présent échappé d'une corne d'abondance, m'avait permis grâce à la faveur de la reine d'équiper trois caravelles, à la grande confusion de mes ennemis. Et après l'ivresse d'une navigation sur des routes inconnues, je connaissais la gloire d'attendre ici, cette nuit, l'apparition d'un soleil qui n'en finissait pas de se lever; peut-être pourrais-je espérer l'immortalité dans la mémoire des hommes, moi qui étais issu du milieu que l'on sait, mais qui pouvais aspirer désormais au titre de Père d'un plus vaste univers... Non, Rodrigo! Tu es baisé ! J'empocherai tes dix mille maravédis de rente !... (p. 108)

Nous remarquons que le morceau que nous avons choisi se compose pour l'essentiel d'une seule grande phrase dont voici le centre: «[...] padecía mi desapego y mi olvido - desapego y olvido debidos [...]». En fait, le centre, c'est un tiret entouré d'une expression qui se répète et qui joue le rôle d'un vase communicant entre les deux parties de la phrase. La première a pour base un segment assez simple: «En cuanto a su renta de diez mil maravedís [...] me la he apropiado ya en beneficio de mi Beatriz [...]». Quant à la deuxième partie, elle a pour base un segment un peu plus complexe et qui enchaîne quatre compléments d'objet direct: «desapego y olvido debidos al Real Favor que sobre mí hubiera derramado [...] la fortuna de tres naves [...], la embriaguez de nuevos rumbos, la gloria de estar aquí [...], y acaso la inmortalidad [...]». Le tout naturellement est truffé de nombreuses incidentes qui font l'accumulation baroque.

On voit que la traduction de Durand est loin de l'écriture carpentiérienne. Il n'est pas exagéré de dire que là où l'on a une seule et longue phrase chez Carpentier, on ne retrouve plus chez le traducteur que des confettis. Pour une unique phrase du texte original, cinq chez le traducteur, et encore l'une d'entre elles est-elle coupée par un point virgule. Que deviennent le rythme, le souffle dans de telles conditions? Et naturellement, les tirets qui ponctuent et organisent la phrase baroque se sont envolés. Sans doute que l'organisation du discours baroque ne fait pas français! La conséquence, c'est que l'oralité, qui est aussi le rythme, et qui se déploie tout au long du passage, en ressort détruite. On le voit aussi dans la suppression des répétitions: «le sé cosas»/《le conviene», «que tarda, que tarda - i cómo tarda», et dans le remplacement de celles-ci par un imparfait: «qui n'en finissait pas de se lever». Le présent oralise, actualise, nous rapproche de Colon, alors que l'imparfait, situant dans le récit, nous en éloigne. C'est toute l'impatience, impatience non dénuée d'une certaine inquiétude, du Découvreur devant sa découverte qui s'exprime par cette répétition et par ce présent. C'est ce que nous dit la signifiance du texte original. Mais pas celle de la traduction.

Il y a aussi la destruction des parallélismes, qui sont une forme de répétition: «reclamando mucho o alborotando más de la cuenta»/ «de trop réclamer ou de faire le malin», où le couple «mucho/más de la cuenta» est estropié. Le recours aux majuscules n'est pas plus prisé du traducteur et disparaît pour: «Mundo», «Quien», et «Real Favor». Les expressions imagées elles non plus ne résistent pas: «Real Favor»/«faveur de la 
reine», pas plus que les métaphores: «Ensanchador del Mundo» / «Père d'un plus vaste univers» ( $\mathrm{sic}$ ), alors que «agrandisseur» ou «élargisseur» sont tout à fait possibles, et que le vocable «Mundo» (avec ou sans majuscule) est l'un des éléments d'une rythmique, qu'il fait partie du mouvement du texte d'un bout à l'autre, qu'il est un élément organisateur du discours, donc forcément du sens. On a encore «anotar en hielo» / «en faire son deuil», alors qu'on a «geler» pour traduire cette métaphore. Il y a aussi des locutions nominales: par exemple «en lágrimas», qui devient un verbe : «pleurait». D'autre part, l'utilisation de l'ajout et de l'explicitation est monnaie courante dans la désécriture : «con la confusión»/ «à la (grande) confusion», «la embriaguez»/ «(Et après) l'ivresse», «de nuevos rumbos»/ «(d'une navigation) sur des routes inconnues», «salido de donde sali» / «issu (du milieu que l'on sait)», «podia aspirar»/ «(mais qui) pouvais aspirer», et «Mundo» / «(d'un plus vaste univers)».

La traduction est tellement occupée à mettre à plat, à remanier, à transformer, bref à défaire le texte, qu'elle en oublie de traduire : «prestas a zarpar», en effet, a disparu. On voit bien que ce qui prime dans le mode de traduire auquel se rattache Durand, c'est le sens. La signifiance, la plupart du temps, n'existe pas. Et ce sens, il est organisé en signifiance dans la traduction suivant des critères classiques et scolaires : phrases courtes, rationalisation, clarification, explicitation des métaphores, etc. Autrement dit, une traduction aveugle à l'écriture. Mais ce qui est particulièrement désolant, c'est qu'une telle traduction, tout en voulant traduire le sens, ne traduit pas le sens, pas tout le sens en tout cas. Car pour cela, encore faudrait-elle qu'elle montre le sujet dans son discours.

Qu'on ait ici en effet une longue phrase n'est pas de peu d'importance sur ce que Carpentier veut donner à comprendre du personnage de Colon. Il y a toute la misère et la gloire de Colon dans ce passage. L'homme et le mythe. C'est en même temps comme un résumé de sa vie, où apparaissent le côté sordide (l'épisode des dix mille maravédis dont il prive injustement Rodrigo de Triana : «i Te jodiste (»), le côté misérable pour l'époque et eu égard à ses ambitions (il a un fils naturel de Beatriz et il est plutôt pauvre). Il y a ensuite la chance qui lui sourit et la réalisation de son entreprise grâce à la Faveur Royale. Et enfin le dédoublement entre l'homme qu'il est, et le mythe qui est là naissant et déjà en train de lui échapper. C'est précisément cela que nous dit la signifiance et que la traduction détruit : «[...] y acaso la inmortalidad, en la memoria de los hombres, de Quien, salido de donde salí, podía aspirar ya al título de Ensanchador del Mundo» (nous soulignons), ce qu'on peut traduire ainsi : «et peut-être l'immortalité, dans la mémoire des hommes de $Q u i$, sorti d'où je suis sorti, pouvait aspirer maintenant au titre d'Élargisseur du Monde». Il y a dans le texte original, dans le travail de l'écriture, une violence faite à la syntaxe, qui s'exprime dans «de Quien, salido de donde salí». Cette violence est le travail de la littérature, qui se fait en marge de la langue, pour créer. Cette violence syntaxique exprime ici que Colon, à la veille de poser le pied sur le sol américain, pressent la naissance d'un autre personnage, son double mythique, qui, étant une troisième personne, Quien, salido, est déjà en train de lui échapper. À cet endroit, on n'a pas à être surpris de l'utilisation d'un pronom relatif comme nom propre, car Carpentier utilise fréquemment ce procédé depuis le début du roman. Ensuite, tout le contraste entre ces deux personnages, toute la grandeur et l'humilité sont résumées dans l'opposition salido (troisième personne)/sali (première personne). Et l'on voit bien que c'est ce nouveau personnage qui va appartenir désormais à l'histoire, qui prend maintenant le pas sur cet homme qu'est Colon, puisque Quien est bien le centre des pensées de l'Amiral, et le sujet grammatical du verbe : podía aspirar ya al título. En outre, la même cuvre de désécriture opère dans la deuxième partie de notre longue phrase, puisque n'apparaît plus la distribution des compléments, dont on a parlé plus haut, et qui dépendent du verbe : hubiera derramado. Ce faisant, c'est l'ensemble de ce 
que doit Colon à la Faveur Royale («tres naves», «la embriaguez de nuevos rumbos», «la gloria», «la inmortalidad»), qui s'en trouve atténué, voire détruit.

On a voulu illustrer à travers cet extrait que les modes de signifier sont intimement liés aux modes d'être. Modes d'être du personnage Colon, de l'écrivain-sujet Alejo Carpentier. La traduction de Durand se situant dans la langue, elle ne connaît pas la littérature. Elle ignore le discours, et donc le sujet et son écriture. Elle est aveugle aux débordements de la langue, c'est-à-dire à ce qui fait que cette écriture se constitue en tant que discours et par là est productrice d'une poétique. Au contraire, la traduction ramène le texte de Carpentier à une simple rhétorique, alors qu'il est tout à fait pertinent, ce que fait Meschonnic, de faire la différence entre langue et discours : «Le travail de la pensée fait une poétique s'il transforme les valeurs de la langue en valeurs de discours, propres à son seul discours. Mais si les catégories de la langue restent des catégories de la langue, c'est le jeu de la rhétorique ${ }^{26}$.» Traduire El arpa y la sombra, c'est donc aussi prendre en compte la poétique du texte. Il y a donc à enfreindre obligatoirement la langue dans la traduction si l'on veut tenir les valeurs du discours carpentiérien. C'est pourquoi il ne faut pas hésiter à traduire «de Quien, salido de donde salí» par le même procédé d'écriture, tout à fait possible en français, possible dans le sens où la signifiance se fait comprendre : «de Qui, sorti d'où je suis sorti». Nous rappellerons que le traducteur se doit d'avoir un projet de traduction qui révèle le texte en tant que système. Ce qui pose le problème de la cohérence de traduction. Ce qui fait toute la différence avec l'antique et dépassé mot à mot. «L'unité n'est pas le mot, mais le texte», et «l'effacement de la signifiance est une occultation de la gestualité, qui est l'oralité organisatrice du texte», dit encore Meschonnic ${ }^{27}$. La gestualité dans El arpa y la sombra, c'est ce que Carpentier invente et qui fait que son texte est discours et littérature.

Le baroquisme est étroitement relié à l'essence latino-américaine. Or, l'accès à l'identité et aux origines passe par une rythmique et par une prosodie qui voyagent dans le roman et dont le rôle, qui consiste à manifester au monde cette essence, a été on ne peut plus nettement défini par l'auteur. Il se trouve que la culture française, de tradition classique, est loin de se sentir en parfaite harmonie avec la culture baroque. C'est pourquoi on peut constater, ce que fait S. Sarduy, un certain refus de cet art si différent, une «résistance morale qu'il (le baroque) a suscité dans certaines cultures de l'économie et de la mesure comme la culture française ${ }^{28} \gg$. Et l'on retombe en cet endroit sur le vieux problème de l'ethnocentrisme dont nous menons la critique. Celle-ci doit donc se dérouler sur deux plans. Sur celui proprement culturel de la vieillerie ethnocentrique, et sur celui de la théorie du langage et de la littérature. C'est de cette façon qu'on fera évoluer les modes de traduire vers le dévoilement et le décentrement que nous appelons de tous nos vœux.

Avec Colon, nous avons un cycle dont il est bientôt l'heure qu'il se referme. Deux dates symboles donc. 1492: la Découverte, le passage du Moyen Âge aux Temps modernes, le début d'un formidable développement du monde occidental et de la constitution des nations européennes qui domineront la planète entière. 1992 : le monde a changé, les nations du vieux continent sont dépassées par des rivalités économiques plus puissantes et sont tentées de créer une Europe unie. Il y a dans ce projet, qui ne saurait se limiter à ses seuls aspects économiques car il n'y a pas d'économie sans culture, une chance à saisir. Car l'altérité est au cœur du problème. Pour construire une Europe qui se tienne, il faudra en passer par la découverte de l'Autre. Et cette chance est double, car aujourd'hui, la construction de l'Europe, c'est aussi, sur une planète internationalisée et qui le sera de plus en plus, son intégration au monde. L'Europe peut et doit montrer la voie de l'Homme décentré. La traduction aussi. Disons qu'elle a là l'occasion de jouer un rôle considérable, et conforme à son histoire faite de la constitution des cultures. Car 
pourquoi ne pourrait-elle pas, après avoir édifié les cultures nationales, montrer l'unicité et en conséquence la culture de l' Homme, par le décentrement?

1492-1992. Pour la traduction, symboliquement, un cycle, donc, dont il y a à sortir. Il faut marquer cette rupture dans les modes de traduire, ce passage de l'ethnocentrisme au dévoilement, par la retraduction de $E l$ arpa y la sombra. Si Colon a découvert l'Amérique, nous avons nous à redécouvrir Colon et les Latino-Américains. Ainsi que le monde. Une telle republication du roman de Carpentier, clairement située dans l'à venir $d u$ monde, serait un signe qu'il est temps que les modes de traduire correspondent à la tâche éthique de la révélation de l'Étranger.

En attendant, pour ne pas faire mentir les propos de cette citation du Zohar: «Les mots ne tombent pas dans le vide» (Las palabras no caen en el vacío), que Carpentier donne en épigraphe, au début de son roman Le Siècle des Lumières, nous donnerons une traduction-dévoilement du demier passage dont nous avons conduit la critique:

Quant à sa rente de dix mille maravédis (et cela certes j'aurai à le dire au confesseur), il pourra noter qu'elle est gelée - et qu'il fasse attention à ne pas aller réclamer excessivement ou à ameuter plus que de raison, vu que sur lui je sais des choses qu'il n'est pas bon pour lui qu'elles se sachent! - parce que cette rente je me la suis déjà appropriée pour le bénéfice de ma Beatriz, la belle Biscaïenne de qui j'ai un enfant sans l'avoir menée à l'autel, et qui, depuis longtemps, en larmes souffrait de mon indifférence et de mon oubli indifférence et oubli dus à la Faveur Royale qui avait versé sur moi, comme jaillie d'une corne d'abondance romaine, la fortune de trois navires prêts à lever l'ancre, en même temps que la confusion de mes ennemis, l'ivresse de nouveaux horizons, la gloire d'être ici cette nuit, dans l'attente du lever d'un soleil qui tarde, qui tarde - et comme il tarde, merde ! - à paraître, et peut-être l'immortalité, dans la mémoire des hommes, de Qui, sorti d'où je suis sorti, pouvait aspirer au titre d'Élargisseur du Monde... Non, Rodrigo ! Tu es baisé ! Je garde tes dix mille maravédis de rente.

Notes

1. Je reprends ici l'orthographe proposée par Todorov, laquelle prend en compte l'intérêt porté par Colon à son nom qui signifie "repeupleur», «colonisateur». Cf. La Conquête de l' Amérique, pp. 32-33.

2. Cf. Cordonnier, L'Homme décentré. Culture et traduction. Traduction et culture (à paraître sous une forme remaniée en 1992). Le présent article est une version abrégée du chap. 6: «Critique d'une traduction».

3. «La femme cachée dans le texte de Kafka», p. 83.

4. Carpentier, La novela latinoamericana en vísperas de un nuevo siglo y otros ensayos, p. 80; c'est l'auteur qui souligne.

5. Ibid., p. 87; «para acabar de entender realmente lo que somos, quiénes somos, y que papel es el que habremos de desempeñar en la realidad que nos circunda y da un sentido a nuestros destinos"; c'est l'auteur qui souligne.

6. Ibid. «Ni el libro europeo, ni el libro yanki, nos darán la clave del enigma hispanoamericano»

7. Ibid.

8. Carlos Fuentes, La nueva novela hispanoamericana, pp. 55-56; «esas pistas magnéticas [...], 'esos sonidos brutos' $[\ldots]$ que [...] integran una nueva totalidad narrativa en la que la ficción se hace a si misma mediante un lenguaje que es reflexión sobre el lenguaje».

9. Titre d'une conférence donnée à Caracas en mai 1975, cf. La novela latinoamericana en vísperas de un nuevo siglo, pp. 111-135.

10. Ibid., p. $114 ;$ «Inventor de palabras, enriquecedor del idioma».

11. Ibid., p. 117; «se caracteriza por el horror al vacio, a la superficie desnuda, a la armonía lineal geométrica, estilo donde en torno al eje central [...] se multiplican lo que podríamos llamar los 'núcleos proliferantes', es decir, elementos decorativos que llenan totalmente el espacio ocupado por la construcción, las paredes, todo el espacio disponible arquitectónicamente, con motivos que están dotados de una expansión propia y lanzan, proyectan las formas con una fuerza expansiva hacia afuera. Es decir es un arte en movimiento, un arte de pulsión, un arte que va de un centro hacia afuera y va rompiendo, en cierto modo, sus propios márgenes.»

12. Ibid., p. 127; "Todo lo insólito, todo lo asombroso, todo lo que se sale de las normas establecidas es maravilloso.» 
13. Ibid., p. 130; «Aqui lo insólito es cotidiano, siempre fue cotidiano.»

14. Ibid., p. 144; «hacer estallar el idioma cuando hace falta».

15. Ibid., p. 181 ; «es el acontecimiento más importante de la historia. Porque existe en la historia universal un hombre anterior al descubrimiento de América, y un hombre posterior».

16. Sur l'œuvre de Carpentier, et plus particulièrement sur La Harpe et l'ombre, cf. la revue Sud: Alejo Carpentier et son cuvre.

17. Cf. Gérard Dufour, «Le viol de Clio», in Sud, p. 109.

18. «La Harpe et l'ombre: signification d'une structure», Sud, pp. 188-189.

19. «En el arpa, cuando resuena, hay tres cosas: el arte, la mano y la cuerda. En el hombre: el cuerpo, el alma y la sombra.»

20. «... contar las cosas como sucedieron, sino como debieron o pudieron haber sucedido.» Commentaire de Carpentier au dos de la couverture de l'édition de 1979.

21. «Mythification et démystification dans La Harpe et l'ombre de A. Carpentier», Sud, p. 236.

22. Cf. André Saint-Lu, «De quelques libertés du romancier avec l'Histoire à propos de El harpa y la sombra de A. Carpentier», in Les Langues néo-latines, $\mathrm{n}^{\circ} 235,1980$, pp. 68-77. Du même auteur, cf. aussi «La Harpe et l'ombre. Roman et Histoire», in Sud, pp. 90-102. Harss, p. 74. «olemos la biblioteca y el archivo».

24. Alors la traduction chantera, p. 75.

25. Émission Tire ta langue, France Culture, 17 mars 1986.

26. «Poétique d'un texte de philosophe et de ses traductions: Humboldt, sur la tâche de l'écrivain et de l'histoire», p. 191.

27. «La femme cachée dans le texte de Kafka», p. 94 et p. 96.

28. El barroco y el neobarroco, p. 176; «resistencia moral que ha suscitado (el barroco) en ciertas culturas de la economía y la mesura como la francesa»; c'est l'auteur qui souligne.

BIBLIOGRAPHIE (La date est celle de l'édition citée.)

CARPENTIER, Alejo (1986) : El arpa y la sombra, Mexico, Siglo veintiuno editores, 8e éd., 1980, traduit de l'espagnol par René L. F. Durand, Paris, Gallimard, coll. «Folio», no 1742.

CARPENTIER, Alejo (1981): La novela latinoamericana en vísperas de un nuevo siglo y otros ensayos, Mexico, Siglo veintiuno editores.

CORDONNIER, Jean-Louis (1989): L'Homme décentré. Culture et traduction. Traduction et culture, thèse de doctorat de $3^{\mathrm{e}}$ cycle, Université de Franche-Comté, Besançon.

FUENTES, Carlos (1980): La nueva novela hispanoamericana, Mexico, Cuadernos de Joaquín Mortiz

HARSS, Luis (1977): Alejo Carpentier o el eterno retomo, in Los nuestros, Buenos Aires, Editorial Sudamericana, pp. 51-86.

MESCHONNIC, Henri (1985): «La femme cachée dans le texte de Kafka», Texte, no $4:$ Traduction/Textualité - Text/Transiatability, pp. 83-98.

MESCHONNIC, Henri (1985): «Poétique d'un texte de philosophe et de ses traductions: Humboldt, sur la tâche de l'écrivain de l'histoire», Les Tours de Babel, Mauvezin, Éditions Trans-Europ-Repress, pp. 181229.

MESCHONNIC, Henri (1987): «Alors la traduction chantera», Revue d'esthétique, nouvelle série, no 12 : La Traduction, Toulouse, éditions Privat, pp. 75-90.

SARDUY, Severo (1982): El barroco y el neobarroco, in América Latina en su literatura (ouvrage collectif), Mexico, Siglo veintiuno editores /UNESCO, Serie América Latina en su cultura, 8e éd., pp. 167-184.

Sud: La Traduction. Réflexion. Reflets, (1987), nos 69-70, Marseille, Diffusion Ulysse, Distribution Distique.

TODOROV, Tzvetan (1982): La Conquête de l'Amérique. La Question de l'Autre, Paris, Seuil. 